\title{
Ambulatory urodynamic monitoring assessment of dorsal genital nerve stimulation for suppression of involuntary detrusor contractions following spinal cord injury: a pilot study
}

\author{
Sean P. Doherty $\mathbb{D}^{1,2} \cdot$ Anne Vanhoestenberghe ${ }^{1} \cdot$ Lynsey D. Duffell $^{1} \cdot$ Rizwan Hamid $^{2} \cdot$ Sarah L. Knight ${ }^{2}$
}

Received: 27 February 2020 / Revised: 27 March 2020 / Accepted: 28 March 2020

(c) The Author(s), under exclusive licence to International Spinal Cord Society 2020

\begin{abstract}
Study design A prospective interventional pilot study using within-individual comparisons.

Objectives To assess the effect of dorsal genital nerve stimulation (DGNS) on urine-storage parameters in participants with spinal cord injury (SCI) and neurogenic detrusor overactivity (NDO) during natural bladder filling.

Setting The London Spinal Cord Injuries Centre at the Royal National Orthopaedic Hospital, Stanmore, UK.

Methods Ambulatory urodynamic monitoring (AUM) was carried out with and without DGNS, before and after a week of using DGNS at home. DGNS was applied on-demand by four participants with bladder sensation, and both continuously and intermittently by one participant with absent sensation. A Wilcoxon sign-rank test was used to test paired results of changes within an AUM session.

Results Urodynamic outcomes were improved using DGNS. Bladder capacity was increased from $244 \pm 59$ to $346 \pm 61 \mathrm{ml}$ ( $p=0.0078$ ), a mean change of $46 \pm 25 \%$. Maximum detrusor pressure was decreased from $58 \pm 18$ to $47 \pm 18 \mathrm{cmH}_{2} \mathrm{O}$ ( $p=0.0156$ ), a change of $17 \pm 13 \%$, and average peak detrusor pressure was decreased from $56 \pm 16$ to $31 \pm 128 \mathrm{cmH}_{2} \mathrm{O}$ $(p=0.0156)$, a mean reduction of $50 \pm 19 \%$. There was an increase in the number of detrusor contractions from the first involuntary detrusor contraction to a strong desire, urgency or incontinence, from $1.5 \pm 1.4$ to $4.3 \pm 1.7$, and an increase in time of $23 \pm 22 \mathrm{~min}$. There were no changes in baseline outcomes following home use of DGNS.

Conclusions DGNS may be applied on-demand, intermittently or continuously, to increase bladder capacity, decrease storage pressures and provide extra time. Improvements were made in addition to existing antimuscarinic medication regimes.
\end{abstract}

\section{Introduction}

Spinal cord injury (SCI) causes permanent damage to motor and sensory function below the level of injury, and profoundly affects the neural control of the lower urinary tract (LUT). Specifically, suprasacral SCI disrupts communication between supraspinal and lumbosacral micturition control centres, leading to an aberration of pathways coordinating the detrusor and sphincters, resulting in

Sean P. Doherty

sean.doherty.15@ucl.ac.uk

1 Aspire Centre for Rehabilitation Engineering and Assistive Technology, University College London, London, UK

2 London Spinal Cord Injury Centre, Royal National Orthopaedic Hospital, London, UK detrusor sphincter dyssynergia (DSD), and the replacement of a spino-bulbo-spinal micturition reflex with a spinal micturition reflex that is no longer modulated consciously, neurogenic detrusor overactivity (NDO). NDO is characterised by involuntary detrusor contractions, often at low volumes. NDO and DSD cause high detrusor pressures, feelings of urgency in those with sensation, episodes of urinary incontinence and incomplete emptying despite leakage. NDO may trigger autonomic dysreflexia in people with injury above $\mathrm{T} 6$ level and increases the risk of ureteral reflux.

The main goal of urological management is to enable low pressure storage of urine and low pressure, efficient voiding to take place at a socially convenient time. This problem is complicated by the combined loss of mobility, loss of sensory feedback from the LUT and loss of voluntary LUT control. Existing treatment methods include oral antimuscarinic medication (AM), intradetrusor injection of 
botulinum toxin-A (BTX) and more recently $\beta-3$ agonist medication such as mirabegron [1], alongside more invasive surgical options such as cystoplasty.

Neuromodulation presents an alternative approach to managing the LUT post SCI. Transcutaneous electrical stimulation of the dorsal genital nerve (DGNS), an afferent branch of the pudendal nerve, has been shown in multiple urodynamic experiments to acutely suppress involuntary detrusor contractions and significantly increase bladder capacity [2,3]. DGNS appears to work by activating the urethral sphincter and bladder neck [4] alongside inhibition of parasympathetic pathways and excitation of detrusorinhibiting sympathetic pathways [5]. This is known to work at a spinal level, in people with complete spinal cord transection, where other neuromodulation sites such as tibial nerve stimulation do not produce the comparable results [6].

Neuromodulation has been applied in several distinct protocols, each with success, where the aim of noncontinuous approaches is to reduce stimulation time: to preserve battery life, improve comfort and reduce the chance of habituation.

On-demand stimulation, used for the immediate suppression of involuntary detrusor contractions, relies on preserved sensation of bladder activity. Following SCI, a majority of people have at least partially preserved bladder sensation, and many use this sensation in their existing bladder management strategy [7]. It is therefore not surprising that positive results have been reported in the small number of pilot studies trialling on-demand DGNS [8-11]. However, issues remain with the assessment of the suitability of residual sensation and also in the accessibility of stimulation triggers to allow fast application of DGNS $[12,13]$. The safety of using partially preserved sensation must also be investigated, as a missed detrusor contraction may lead to ongoing high intradetrusor pressures.

For those without residual sensation, in the absence of a physiological trigger for conditional stimulation, the options are continuous or intermittent stimulation. Continuous stimulation has been shown to be effective during standard cystometry [2] and in a limited number of patients (4) over longer periods (between 4 and 8 weeks) [8, 14]. Intermittent DGNS has been shown to be effective in one standard cystometry study of five SCI participants, where a 5-s on, 5 -s off regime was trialled during slow $(10 \mathrm{ml} / \mathrm{min})$ fill cystometry, increased the maximum capacity by $120 \mathrm{ml}$, comparable with a $135 \mathrm{ml}$ increase seen during continuous DGNS [15].

The majority of trials of DGNS have looked at the acute effect of conditional, continuous or on-demand stimulation during standard cystometry, filling the bladder at nonphysiological rates [2, 3, 16-18]. Yet for appropriate triggering of DGNS, Martens et al. [12] found a large disparity in the ability to detect onset of NDO between standard CMG and natural fill CMG in participants with SCI and residual bladder sensation. It is potentially safer, therefore, to test the adequacy of a person's residual sensation for triggering DGNS using ambulatory urodynamic monitoring (AUM), to prevent unmonitored use of on-demand stimulation where periods of high pressure go undetected.

We aimed to trial on-demand, continuous or intermittent DGNS protocols, selected as appropriate for individual people with SCI depending on residual bladder sensation, using AUM to assess the effect on bladder pressure and capacity. Further to this, in previous trials of DGNS at home, gains have been made in baseline CMG outcomes whilst using DGNS for periods as short as 3 days [10]. We aimed to assess baseline AUM outcomes, without the application of DGNS, before and after a 7-day period of DGNS use in participants homes.

\section{Materials and methods}

We certify that all applicable institutional and governmental regulations concerning the ethical use of human volunteers were followed during the course of this research.

\section{Participants}

Five people with chronic suprasacral SCI and urodynamically proven NDO participated in this study. Four out of the five participants had retained sensation, defined as sensation used in their daily lives for timing of bladder voiding. The mean age was $52 \pm 11$ years old, and time from injury $12 \pm 5$ years. All participants were on some form of AM, which continued over the study period, and $4 / 5$ had previously undergone BTX injections (all more than 6 months prior to participation). Four out of five participants voided by using intermittent self catheterisation (ISC) and one reflex voided into a sheath drainage system. All participants reported experiencing incontinence of varying regularity.

\section{Stimulation system and set-up}

An Odstock Medical Ltd 'Pace' one-channel stimulator was used with custom wireless, bluetooth low energy (BLE), controller and Android smartphone app to control the stimulation [19]. For participants with residual bladder sensation, direct (on/off) control of stimulation from the app was enabled and they triggered stimulation at first sensation of urge and turned it off when any sensation of urge or NDO subsided. For P01, who had no bladder sensation, two stimulation modes were set up. (i) Direct control for continuous stimulation, and (ii) an automatic stimulation 
A)

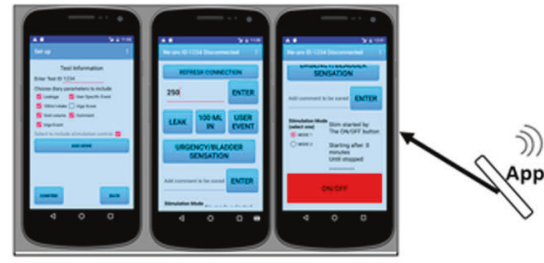

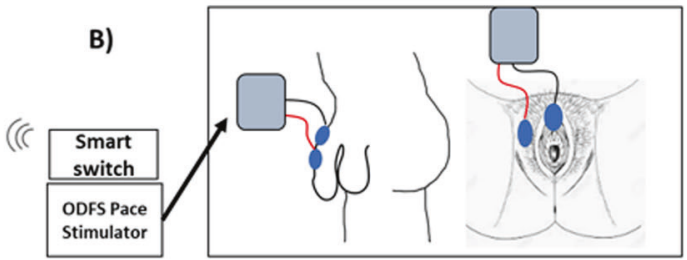

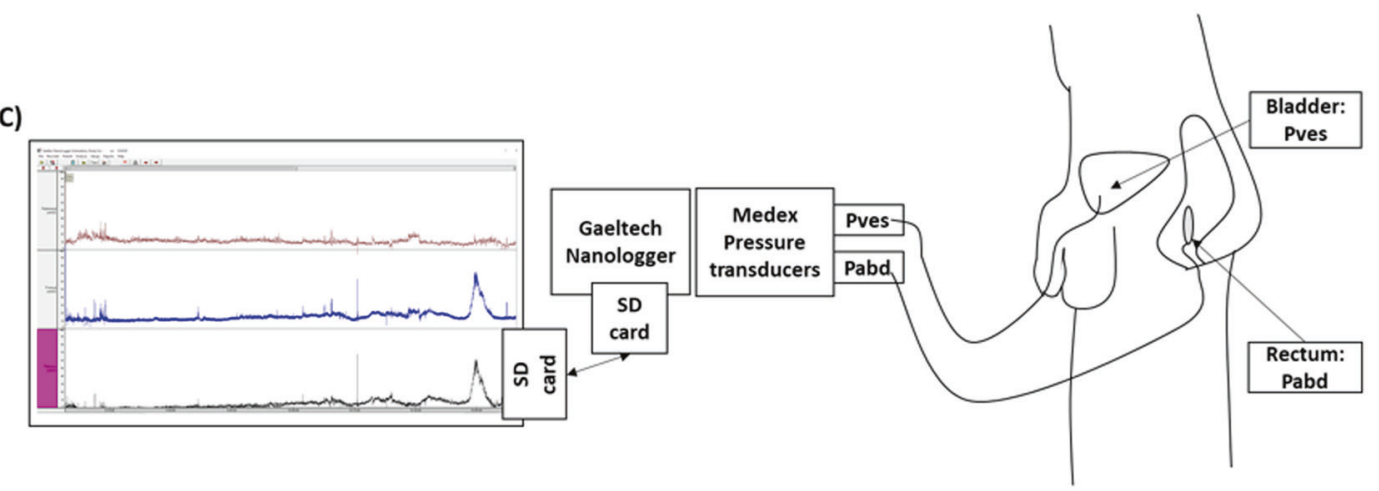

Fig. 1 Equipment set-up. a Custom Android app used for control of the stimulation device. b Sketch of electrode positioning and connection to the pace stimulator, connected to the smartphone via

sequence (alternating $5 \mathrm{~s}$ on and $5 \mathrm{~s}$ off) to be initiated by participants as an alternative to continuous stimulation.

Stimulation was biphasic, $200-\mu \mathrm{S}$ wide, constant voltage pulses delivered at $15 \mathrm{~Hz}$. The stimulation amplitude was set during the participants' first visit to the clinic. This was done by visual detection of external anal sphincter response $\left(\mathrm{EAS}_{\text {thresh }}\right)$ to increasing stimulation amplitudes. The amplitudes used in the study were then set as close to twice $\mathrm{EAS}_{\text {thresh }}$ as possible. Based on empirical findings of improved participant comfort, a 2-s ramp at the initiation of stimulation was set on the stimulator.

PALS platinum $2.5-\mathrm{cm}$ round electrodes were used, placed on the penis or clitoris as shown in Fig. 1. Male participants placed electrodes on the dorsum of the penis, $\sim 2 \mathrm{~cm}$ apart with the cathode (black lead) placed proximal to the anode, and female participants were instructed to place the cathode over the clitoris and the anode to one side on the labia majora or inner thigh.

\section{Ambulatory urodynamic monitoring (AUM)}

AUM involves continuous measurement of vesical $\left(P_{\mathrm{ves}}\right)$ and abdominal $\left(P_{\mathrm{abd}}\right)$ pressures during physiological filling cycle, calculating detrusor pressure $P_{\text {det }}=P_{\text {ves }}-P_{\text {abd }}$. In contrast to the filling $\mathrm{CMG}$ performed in many previous studies of DGNS, AUM records bladder activity in a close to real life scenario, where the patient is able to move around freely [20] and the bladder is filled naturally with urine, rather than retrogradely with saline. bluetooth low energy. c Schematic of AUM set up with an example trace seen on the computer at the end of a filling cycle.

Pressures were recorded using a commercial data logging system (Nanologger, Gaeltech Devices Ltd, Isle of Skye, Scotland), with commercial pressure transducers zeroed at atmospheric pressure (Medex Logical, Smiths Medical Ltd, USA). To enable multiple filling cycles, we used a $10 \mathrm{Ch}$ indwelling Foley urethral catheter to allow emptying and pressure recordings to be done in one secure line. Abdominal pressure was recorded through a water-filled balloon catheter placed in the rectum.

Participants were invited to attend our centre for AUM twice, 7 days apart. Between these two dates, participants were given the stimulation device to use at home. During AUM, participants were able to move freely and were encouraged to drink. Each AUM session began with a natural filling cycle (control), before a second natural filling cycle where DGNS was used. In one session, we had time for a further filling cycle where P01 trialled intermittent DGNS, set to a $5 \mathrm{~s}$ on/off regime.

\section{Data analysis}

The urodynamic data were analysed in Gaeltec Nanologger software. Further analysis was conducted using Matlab. Average peak detrusor pressure (APDP), maximum detrusor pressure (MDP) and maximum cystometric capacity (MCC), defined as the volume withdrawn at the end of the test, including leakage, captured using pad weight, were recorded, and changes from the baseline were analysed within individuals. Stimulation triggering was recorded on 


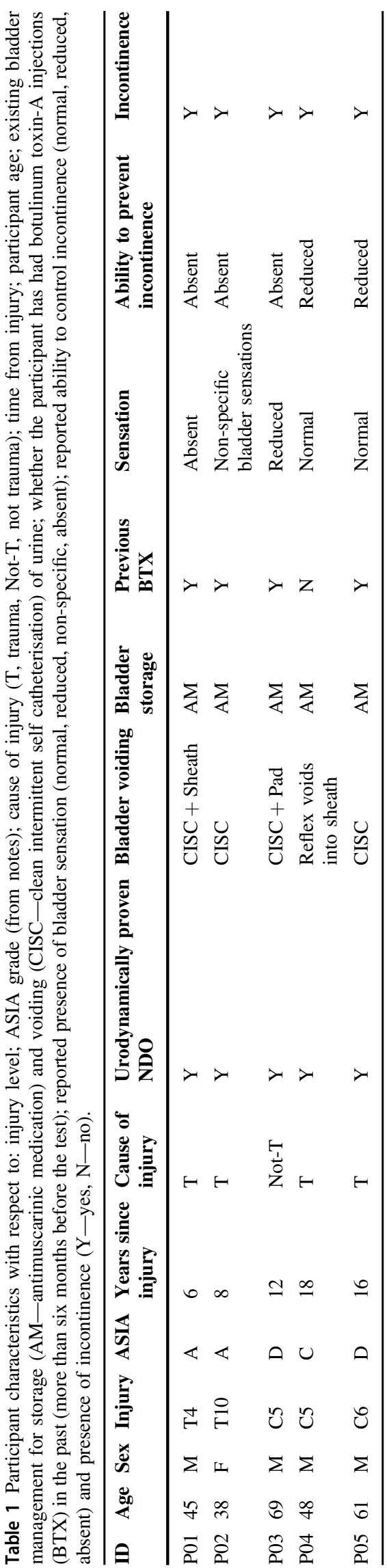

the Android app as a timestamped event with the clock synced to the Gaeltec device.

All statistical analysis was completed using Matlab (Mathworks, version 2017b) and Excel (Microsoft, version Mac 15.24). A Wilcoxon signed-rank test was used to test matched pairs of baseline and with stimulation measurements from the same session, where $p<0.05$ was considered significant. All values unless otherwise stated are reported as mean \pm standard deviation.

\section{Results}

Demographic data for the five participants are shown in Table 1; ISNCSCI level and degree of injury are unavailable. All five participants attended at least one AUM session. $\mathrm{P} 02$ and $\mathrm{P} 03$ were unable to attend and complete the second AUM session, respectively, for reasons unrelated to the intervention. In addition, P03's AUM pressure data in week 1 was not recorded as a wire was knocked out of place during ambulation, therefore only the data on MCC were recorded. All AUM outcomes are shown in Table 2.

\section{AUM results}

During AUM, MCC was increased from $244 \pm 59$ to $346 \pm$ $61 \mathrm{ml}$ when using DGNS ( $p=0.0078)$, a mean change from control of $102 \pm 46 \mathrm{ml}$ or $46 \pm 25 \%$. Individual results are shown in Fig. 2. The time from first detrusor contraction to either leakage or strong desire to void was $6 \pm 13 \mathrm{~min}$ at baseline and $28 \pm 16 \mathrm{~min}$ with DGNS $(p=0.0469)$, increased by $23 \pm 22 \mathrm{~min}$.

MDP was $58 \pm 18 \mathrm{cmH}_{2} \mathrm{O}$ in control and $47 \pm 18 \mathrm{cmH}_{2} \mathrm{O}$ with DGNS $(p=0.0156)$, reduced by $10 \pm 9 \mathrm{cmH}_{2} \mathrm{O}$ or $17 \pm 13 \%$. APDP was reduced from a control value of $56 \pm$ $16 \mathrm{cmH}_{2} \mathrm{O}$ to $31 \pm 18 \mathrm{cmH}_{2} \mathrm{O}$ with DGNS ( $p=0.0156$ ), a reduction of $27 \pm 14 \mathrm{cmH}_{2} \mathrm{O}$ or $50 \pm 19 \%$.

DGNS suppressed involuntary detrusor contractions in all participants, enabling previously terminal NDO to become lower pressure contractions of short duration. Therefore, the number of detrusor contractions, before leakage or urgency requiring emptying, increased from $1.5 \pm 1.4$ during control to $4.3 \pm 1.7$ when using DGNS $(p=0.0234)$. Continuous and intermittent stimulation were used by P01, with both, low amplitude detrusor contractions were seen, not resulting in leakage.

P01 and P03 were the only participants who experienced leakage during the test, P01 was the only participant with absent sensation and was only aware of needing the toilet when he was wet, the filling cycle was considered as over after leakage had occurred both in baseline and with DGNS cycles. P03 leaked during the baseline, but not during the DGNS trial. All other participants 


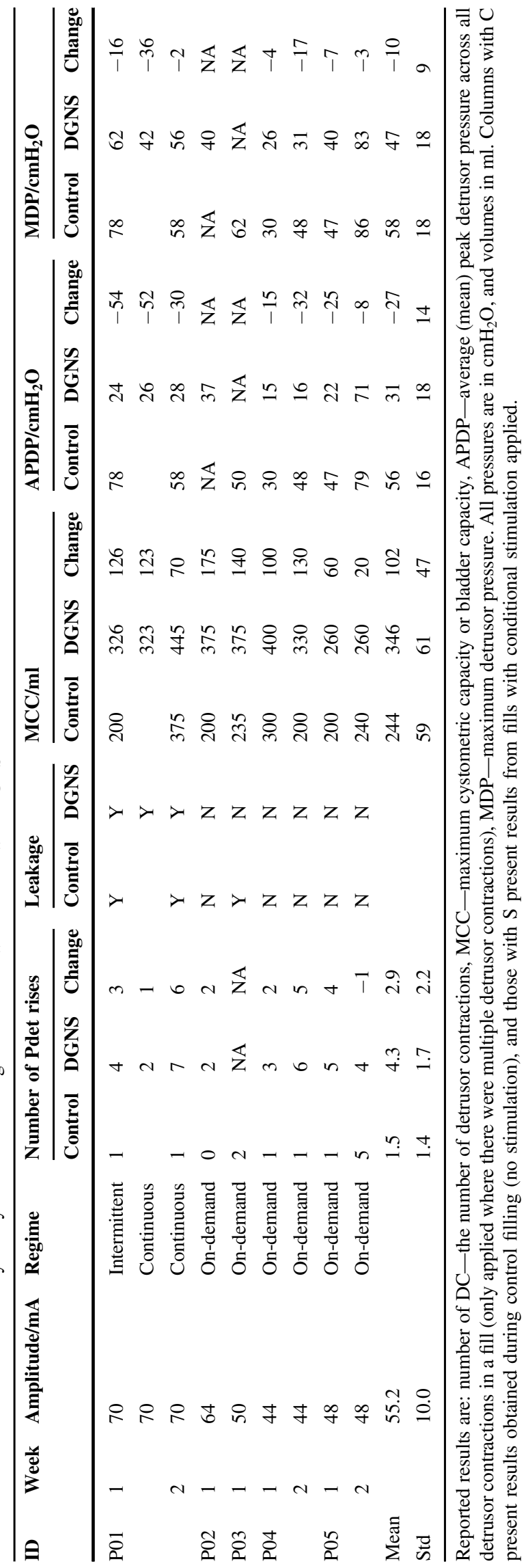

terminated the test when they felt a strong desire to void, prior to leakage occurring.

\section{Stimulation use at home}

During the 7-day period at home, DGNS was used by participants $29 \pm 19$ times over the week, range 8-54 times. It was used on-demand by four participants and in continuous mode by one. Stimulation was used just eight times by $\mathrm{P} 01$, though in continuous mode for a period of $84 \pm$ $58 \mathrm{~min}$.

\section{Discussion}

Five published studies have trialled DGNS to suppress involuntary detrusor contractions, present following SCI, in participants' homes $[8-11,14]$. These have included a total of 25 participants with SCI, 19 of whom completed the respective studies. Four of the 19 participants used a continuous stimulation paradigm $[8,14]$, the remaining 15 triggered stimulation on urge [8-11]. Six of the 15 who used 'on urge' stimulation trialled semi-conditional stimulation, where an automatic on-off regime of stimulation persisted after the user had triggered on urge, and 9/15 used stimulation controlled manually (on urge). Across all studies, 9 of the 19 were on AM during the study. Our pilot study addresses the gap in the data of how DGNS effects urodynamic parameters when applied in a manner comparable with home-based trials: during natural filling and using on-demand or continuous stimulation regimes. We report that neuromodulation, in addition to existing antimuscarinic medication regimes, was able to reduce peak storage pressures and increase bladder capacities during ambulatory urodynamic monitoring. This adds to the limited data currently available on the use of DGNS in a chronic setting and supports the efficacy of DGNS as an adjunctive method of improving management of NDO.

\section{Retained sensation was adequate for triggering neuromodulation}

Following SCI, sensation of the bladder is severely compromised. However, a majority of all those with SCI do maintain some sensation or awareness, even a significant minority of those with clinically complete injuries [7], that may be suitable for triggering neuromodulation. An important point to assess is whether the retained sensation is specific or accurate enough to detect the onset of NDO sufficiently quickly to trigger DGNS to maintain continence and maintain low bladder pressures. This has been shown to be the case during filling cystometry and home use of DGNS. However, important data comparing detection of 

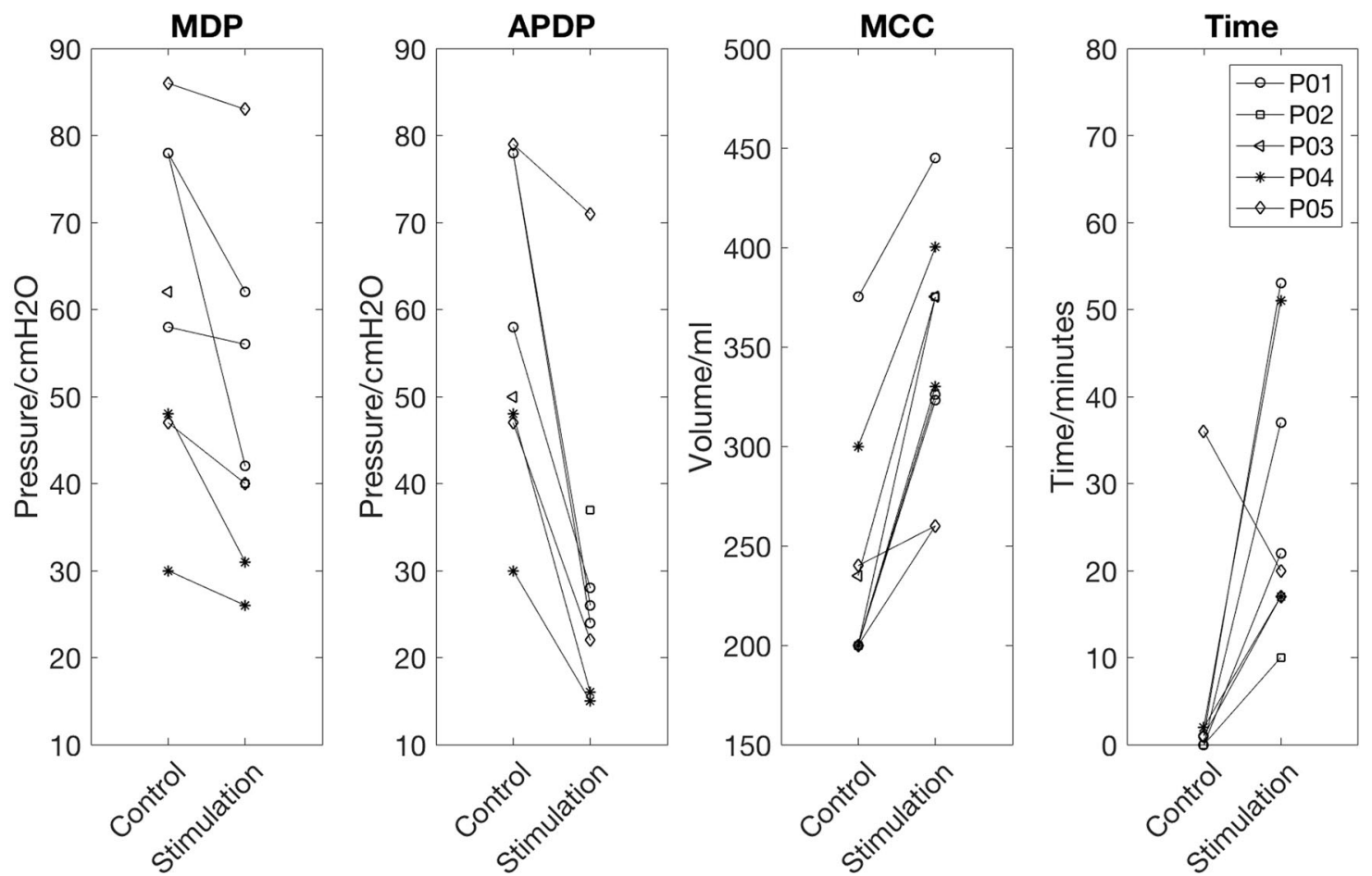

Fig. 2 Individual results from AUM recordings across all sessions without (control) and with (Stimulation) DGNS. From left to the right: maximum detrusor pressure (MDP) can be seen to decrease in all available recordings, including for $\mathrm{P} 01$ where MDP was also leak point pressure. Average Peak detrusor pressure (APDP), another measure of pressure within the bladder relevant to a treatment where multiple spikes in pressure are expected (as with on-demand DGNS), decreases were seen in all recordings. Maximum cystometric capacity

bladder pressure rise during filling cystometry versus during natural filling (AUM) showed that significantly less detrusor contractions were detected by participants during AUM [12]. During this study, we assessed the ability of participants who reported having bladder sensation to correctly trigger stimulation to reduce bladder pressures. In the small number assessed, ranging AIS A-D, we showed that stimulation was able to be triggered appropriately, as denoted by the large drop in average peak detrusor pressure to $31 \pm$ $18 \mathrm{cmH}_{2} \mathrm{O}$, a mean individual decrease of $50 \%$.

Hansen et al. [17] used automatically triggered DGNS at a rise of $10 \mathrm{cmH}_{2} \mathrm{O}$, in a similar SCI group of 16 who had not ceased AM regimes and were assessed during natural filling of the bladder. The results showed a similar decrease in APDP, of $58 \%$ from $72 \pm 28$ to $28 \pm 13 \mathrm{cmH}_{2} \mathrm{O}$, with a bladder capacity increase of $53 \pm 36 \%$. Our results, whilst limited by number, suggest that on-demand stimulation is similarly effective to pressure-driven conditional neuromodulation in appropriately selected people with SCI, during natural filling. This is also seen during standard cystometry [16].

There are several potential advantages to using ondemand DGNS for managing NDO, it is important therefore

(MCC) was increased in all participants. Time, in minutes from first involuntary detrusor contraction to end of the test, increased using DGNS in all participants, but not in both sessions for P05, where the participant had several detrusor contractions that neither resulted in leakage or strong desire to void. Time is perhaps the most potent demonstration of how DGNS might be applicable to the daily lives of people living with NDO.

to understand the applicability of this technique across the SCI population and to understand the prevalence and nature of retained bladder sensation following SCI. Potential advantages of on-demand stimulation over other stimulation paradigms are that (i) it reduces stimulation time, thereby reducing power consumption, stimulation 'dose' and any possibility of habituation of reflex inhibition, and (ii) it is not reliant on any physiological trigger in a closed-loop system; therefore, the system may remain completely noninvasive and, should such a trigger become available, it may be integrated into a system without difficulty. Potential advantages of on-demand DGNS over AM or BTX are that it does not appear to have any side effects and is reversible; and it utilises a different approach, where the baseline condition of NDO is not altered, but the user is given control to 'switch off' unwanted detrusor contractions. We do not know whether this approach will have a long-term therapeutic benefit.

These potential advantages must be balanced with disadvantages, which may be reduced with further work. For on-demand DGNS, whilst it allows the flexibility of a noninvasive system and gives control to the user, it requires wearing electrodes on the genitalia, and the engagement of 
the user to trigger stimulation. This is a clear disadvantage when compared with AM where medication is taken once per day or BTX where one injection is required every 6 to 12 months; it is for this reason that we believe showing that DGNS is effective in addition to existing AM regimes is important, as patients may titrate a combined approach depending on daily activities and side effects of AM. It is possible that an implantable device, such as an adapted sacral anterior root stimulator, set-up to stimulate the posterior or mixed Sacral Nerves of S2-4, may be highly effective for patients to be able to use an on-demand stimulation regime, but be free of genital electrodes. This approach was demonstrated in a successful pilot study using continuous stimulation [21].

\section{Time from first NDO to leakage}

One outcome that may be of the most importance to potential users of DGNS is whether it can increase the time between first sensation and urinary leakage, and thus provide control over continence for a long enough period to reach a suitable toilet. Our results during AUM, in four participants, showed an increase of $23 \pm 22 \mathrm{~min}$ in this period, with a range of times from 10 to $53 \mathrm{~min}$. For anyone with either retained sensation or artificial feedback in place, it is a key positive element of DGNS over existing therapies, in that it may be applied on-demand to control continence for a period.

\section{Bladder inhibition in addition to AM}

DGNS was able to suppress involuntary detrusor contractions and increase capacity whilst participants remained on AM medication. Pudendal afferent stimulation effects inhibition via both parasympathetic and sympathetic reflexes $[4,5]$; this may explain the complementary effect of DGNS to AM. It is unclear if this effect would be maintained whilst also on $\beta-3$ agonist medication, targeting the sympathetic innervation of the detrusor.

\section{Change in the baseline (without DGNS) urodynamic parameters following home use of DGNS}

Urodynamic outcome measures before and after a week of using DGNS at home were only obtained from three of the five participants in this study, and no consistent changes were noticed in any parameter. From week 1 to week 2 , there was an increase in baseline bladder capacity in two $(\mathrm{P} 01, \mathrm{P} 05)$, with a decrease in one (P04) and an increase in detrusor pressure in two (P04, P05), with a decrease in one (P01). Our results do not follow the small improvements seen in baseline urodynamic parameters in some other pilot studies where changes have been noted following as little as 3 days of on-demand DGNS use [10]. Larger studies with longer courses of DGNS are required to detect any DGNS related changes in baseline parameters and the duration of any observed carryover effect. Although not specifically measured, no changes were noted in bowel or sexual function in any participant.

\section{Study limitations}

The conclusions of this pilot study are drawn from a small sample size of just five participants, where each participant acted as their own control. Further to this, bladder sensations during natural filling of the bladder and their suitability for triggering NDO could have been better assessed by the inclusion of graded bladder sensation recordings alongside the pressure recordings [22]. Whilst users triggered stimulation based on their sensation (on-demand DGNS), this should be added to future protocols to better capture the escalation of sensations and their relationship to both detrusor pressure and DGNS use.

The wireless control via smartphone app received positive feedback, users with C5 injury were able to trigger stimulation with ease, though the device was found to be a little bulky, and having to charge both the phone and the smart switch was an annoyance.

\section{Conclusions}

The results from this trial indicate that it may be safe and practical to manage NDO following SCI using on-demand, intermittent and continuous DGNS paradigms in addition to existing antimuscarinic medication regimes where NDO is still present. Within-individual results showed increase in time from first involuntary detrusor contraction to strong desire to void or incontinence, with a corresponding increase in bladder capacity. A decrease was found in maximum detrusor pressure, and several detrusor contractions were suppressed using DGNS leading to a reduced average peak detrusor pressure. We found no consistent changes in baseline parameters of pressure and capacity following use of DGNS at home in three participants. To further these results and build on the literature, longer study of DGNS is required. Given the range of bladder sensation or awareness following SCI, it will be important to study the success of on-demand stimulation across a range of people with SCI in longer term, at-home, studies of DGNS. Further to this, it will be important to assess the acceptability and usability of DGNS devices, and where DGNS may fit into existing treatment regimes. For each, continued user engagement is key. 


\section{Data availability}

The data sets generated and analysed during this study are available from the corresponding author on reasonable request.

Acknowledgements We thank The INSPIRE Foundation for funding this project.

\section{Compliance with ethical standards}

Conflict of interest The authors declare that they have no conflict of interest.

Publisher's note Springer Nature remains neutral with regard to jurisdictional claims in published maps and institutional affiliations.

\section{References}

1. Drake MJ, Apostolidis A, Cocci A, Emmanuel A, Gajewski JB, Harrison SCW, et al. Neurogenic lower urinary tract dysfunction: clinical management recommendations of the Neurologic Incontinence committee of the fifth International Consultation on Incontinence 2013. Neurourol Urodynam. 2016;35:657-65. https://doi.org/10.1002/nau.23027

2. Kirkham APS, Shah NC, Knight SL, Shah PJR, Craggs MD. The acute effects of continuous and conditional neuromodulation on the bladder in spinal cord injury. Spinal Cord. 2001;39:420-8. https://doi.org/10.1038/sj.sc.3101177

3. Bourbeau DJ, Creasey GH, Sidik S, Brose SW, Gustafson KJ. Genital nerve stimulation increases bladder capacity after SCI: a meta-analysis. J Spinal Cord Med. 2018;41:426-34. https://doi. org/10.1080/10790268.2017.1281372

4. Reitz A, Schmid DM, Curt A, Knapp PA, Schurch B. Afferent fibers of the pudendal nerve modulate sympathetic neurons controlling the bladder neck. Neurourol Urodynam. 2003;22:597-601. https://doi.org/10.1002/nau.10134

5. Lindström S, Fall M, Carlsson CA, Erlandson BE. The neurophysiological basis of bladder inhibition in response to intravaginal electrical stimulation. J Urol. 1983;129:405-10. https://doi. org/10.1016/S0022-5347(17)52127-8

6. Doherty SP, Vanhoestenberghe A, Duffell LD, Hamid R, Knight SL. A urodynamic comparison of neural targets for transcutaneous electrical stimulation to acutely suppress detrusor overactivity following spinal cord injury. Fron. Neurosci. 2019;13. https://doi. org/10.3389/fnins.2019.01360

7. Ersoz M, Akyuz M. Bladder-filling sensation in patients with spinal cord injury and the potential for sensation-dependent bladder emptying. Spinal Cord 2004;42:110-6. https://doi.org/10. 1038/sj.sc. 3101525

8. Bourbeau DJ, Gustafson KJ, Brose SW. At-home genital nerve stimulation for individuals with SCI and neurogenic detrusor overactivity: a pilot feasibility study. J Spinal Cord Med. 2018;11:1-11. https://doi.org/10.1080/10790268.2017.1422881
9. Lee Y-H, Creasey GH. Self-controlled dorsal penile nerve stimulation to inhibit bladder hyperreflexia in incomplete spinal cord injury: a case report. Arch Phys Med Rehabilitation. 2002;83:273-7. https://doi.org/10.1053/apmr.2002.28817

10. Opisso E, Borau A, Rijkhoff NJM. Subject-controlled stimulation of dorsal genital nerve to treat neurogenic detrusor overactivity at home. Neurourol Urodynam. 2013;32:1004-9. https://doi.org/10. 1002/nau.22359

11. Lee YH, Kim SH, Kim JM, Im HT, Choi IS, Lee KW. The effect of semiconditional dorsal penile nerve electrical stimulation on capacity and compliance of the bladder with deformity in spinal cord injury patients: a pilot study. Spinal Cord. 2012;50:289-93. https://doi.org/10.1038/sc.2011.141

12. Martens FMJ, van Kuppevelt HJM, Beekman JAC, Rijkhoff NJM, Heesakkers JPFA. Limited value of bladder sensation as a trigger for conditional neurostimulation in spinal cord injury patients. Neurourol Urodynam. 2010;29:395-400. https://doi.org/10.1002/ nau. 20770

13. van Breda HMK, Farag FF, Martens FMJ, Heesakkers JPFA, Rijkhoff NJM. Subject-controlled, on-demand, dorsal genital nerve stimulation to treat urgency urinary incontinence; a pilot. Front Neurosci. 2016;10:24. https://doi.org/10.3389/fnins.2016.00024

14. Wheeler JS, Walter JS, Sibley P. Management of incontinent SCI patients with penile stimulation: preliminary results. J Am Paraplegia Soc. 1994;17:55-9.

15. Zafirakis H, Knight S, Shah PJR, Balasubramaniam V, Kirkham APS, Hamid R. et al. Intermittent versus continuous electrical stimulation of the dorsal penile nerve on bladder capacity in spinal cord injury. Spinal Cord. 2001;39:420-8.

16. Opisso E, Borau A, Rodriguez A, Hansen J, Rijkhoff NJM. Patient controlled versus automatic stimulation of pudendal nerve afferents to treat neurogenic detrusor overactivity. J Urol. 2008;180:1403-8. https://doi.org/10.1016/j.juro.2008.06.023

17. Hansen J, Media S, Nøhr M, Biering-Sørensen F, Sinkjaer T, Rijkhoff NJM. Treatment of neurogenic detrusor overactivity in spinal cord injured patients by conditional electrical stimulation. J Urol. 2005;173:2035-9. https://doi.org/10.1097/01.ju.0000158160. $11083.1 \mathrm{~b}$

18. Wheeler JS, Walter JS, Zaszczurynski PJ. Bladder inhibition by penile nerve stimulation in spinal cord injury patients. J Urol. 1992;147:100-3. https://doi.org/10.1016/s0022-5347(17)37145-8

19. Doherty SP, Knight SL, Vanhoestenberghe A. Developing a wireless device for the research of practical neuromodulation techniques to treat the neurogenic bladder. In: Proceedings of International Functional Electrical Stimulation Society Conference, Montpellier, France, 2016.

20. van Waalwijk van Doorn E, Anders K, Khullar V, KulsengHanssen S, Pesce F, Robertson A, et al. Standardisation of ambulatory urodynamic monitoring: Report of the Standardisation Subcommittee of the International Continence Society for Ambulatory Urodynamic Studies. Neurourol Urodynam. 2000;19:113-25.

21. Kirkham APS, Knight SL, Craggs MD, Casey ATM, Shah PJR. Neuromodulation through sacral nerve roots 2 to 4 with a Finetech-Brindley sacral posterior and anterior root stimulator. Spinal Cord. 2002;40:272-81.

22. Craggs MD. Objective measurement of bladder sensation: use of a new patient-activated device and response to neuromodulation. BJU Int. 2005;96:29-36. https://doi.org/10.1111/j.1464-410X.2005.05649.x 